\title{
An Improved Control Scheme for Grid-forming Inverters
}

\author{
Yemi Ojo, Jeremy Watson, and Ioannis Lestas \\ Department of Engineering \\ University of Cambridge \\ Cambridge, United Kingdom \\ yo259@cam.ac.uk,jdw69@cam.ac.uk, ic120@cam.ac.uk
}

\begin{abstract}
In order to reduce the reliance of power grids on conventional (and often non-renewable) generation, reliable and dispatchable converter-interfaced distributed generators (DGs) are required. Instead of relying on large rotating machines for frequency and voltage regulation, it becomes crucial to develop improved control schemes for grid-forming inverters. In this paper we propose a simple and effective frequency and voltage control scheme that offers desirable dynamic response and power sharing. The proposed controller is compared to the conventional hierarchical control scheme via a stability analysis of the overall system dynamics and time-domain simulation results. It is shown that the transient performance and the stability properties are significantly improved.
\end{abstract}

Index Terms-Microgrid, grid-forming inverter, grid-forming control, small-signal analysis.

\section{INTRODUCTION}

To reduce greenhouse gas emissions and make electrical power readily available and accessible, renewable energy sources (RESs) are being explored and utilised. These are incorporated into existing power systems through inverterbased microgrids, which consist of distributed generators (DGs). While grid-following operation of inverters is easy to implement, reliance on non-renewable conventional grids for voltage and frequency reference prevents autonomous operation, a key feature of microgrids. To remedy this, reliable and dispatchable converter-interfaced DGs are required, and hence, becomes crucial to develop improved control schemes for grid-forming inverters.

Several control approaches have been developed for gridforming inverters. Conventional droop control approaches equip inverters with similar behaviour to synchronous generators. This includes frequency and voltage droop control [1], angular droop control [2], and virtual impedance control [3], [4]. The matching control [5], involves augmenting the inverter dynamics with an internal oscillator that sets frequency via the tracking of a desired DC voltage. The virtual oscillator approaches control converters as nonlinear limit cycle oscillators [6], [7]. In comparison to other schemes, droop control approaches offer robustness, simplicity of implementation, and power sharing in parallel inverters without physical communication links [1]. The disadvantage of the conventional frequency controllers is the associated nonlinearities through the power relations.

A common control approach in inverters is the implementation of conventional droop with strict hierarchical inner (current) and outer (voltage) control loops [8]-[10]. Such control schemes introduce lags/delays and nonlinearities in the generation of the control input to the pulse-width modulating

This work was supported by ERC starting grant 679774 .
(PWM) block [11]. These lags/delays reduce stability margins and can often lead to oscillatory responses [2], [11]. The study of control schemes that can provide improved performance is therefore of interest. A frequency and voltage controller that provides appropriate passivity properties was proposed in [12]. In this paper we investigate an alternative simple frequency and voltage control scheme that offers a desirable transient performance and stability margin. In particular, we compare its performance to that of conventional hierarchical control, via a small-signal stability analysis of the overall system dynamics, and also carry out time-domain simulations on advanced models.

The main contributions of this paper are as follows:

1) As opposed to conventional droop control with cascaded inner (current) and outer (voltage) controllers, we propose a simple frequency and voltage control scheme which eliminates the nonlinearities associated with conventional control schemes, achieve better power sharing response, has current limiting capability, and is implemented with a single inner voltage loop to reduce lags/delays and oscillatory responses.

2) We perform a stability analysis and numerical study to demonstrate the performance of the proposed controllers compared to the conventional controllers.

The paper is organized as follows. Preliminaries and notation are introduced in section II, which includes basic definitions, and a description of the inverter and load models. The conventional and proposed control schemes are described in section III. The comparative study of the proposed and conventional controllers are presented in section IV, which includes a stability analysis and time domain simulations. Finally, our conclusions are given in section $\mathrm{V}$.

\section{PReliminaries AND Notation}

Let $\mathbb{R}_{\geq 0}:=\{x \in \mathbb{R} \mid x \geq 0\}$, and $\mathbb{R}_{>0}:=\{x \in \mathbb{R} \mid x>$ $0\}$. Let $\bar{x}=\operatorname{col}\left(x_{i}, \ldots, x_{n}\right) \in \mathbb{R}^{n}$ denote a column vector with entries $x_{i} \in \mathbb{R}$, and whenever clear from the context $x=\operatorname{col}\left(x_{i}\right) \in \mathbb{R}^{n} . x^{\top}$ denotes respectively the transpose of $x \in \mathbb{R}^{n}$ and complex transpose of $x \in \mathbb{C}^{n}$. Let $\mathcal{J}=\left[\begin{array}{cc}0 & 1 \\ -1 & 0\end{array}\right]$.

Definition 1 (Symmetric AC three-phase signal): A signal $x_{a b c}: \mathbb{R}_{>0} \rightarrow \mathbb{R}^{3}$ denotes a symmetric three-phase AC signal of the form $x_{a b c}(t)=\operatorname{col}(\hat{X} \sin (\omega t), \hat{X} \sin (\omega t-$ $\left.\frac{2 \pi}{3}\right), \hat{X} \sin \left(\omega t+\frac{2 \pi}{3}\right)$ ) where $\hat{X} \in \mathbb{R}_{\geq 0}, \omega, t \in \mathbb{R}_{>0}$ are respectively the amplitude, frequency and time.

Remark 1: In this paper, three-phase symmetric AC signals of the form described in Definition 1 are considered. 
Definition 2: The Park transformation $T(\omega t): \mathbb{R}_{>0} \times$ $\mathbb{R}_{>0} \rightarrow \mathbb{R}^{3 \times 3}$ we adopt is the power invariant form given by

$$
T(\omega t)=\sqrt{\frac{2}{3}}\left[\begin{array}{ccc}
\sin (\omega t) & \sin \left(\omega t-\frac{2 \pi}{3}\right) & \sin \left(\omega t+\frac{2 \pi}{3}\right) \\
\cos (\omega t) & \cos \left(\omega t-\frac{2 \pi}{3}\right) & \cos \left(\omega t+\frac{2 \pi}{3}\right) \\
\frac{1}{\sqrt{2}} & \frac{1}{\sqrt{2}} & \frac{1}{\sqrt{2}}
\end{array}\right]
$$

Definition 3 (Local Direct-Quadrature Coordinates): The direct-quadrature-zero coordinates in the local reference frame, denoted by the subscript $d q 0$, are obtained when the Park transformation (1) is applied to a three-phase AC signal $x_{a b c}$ with frequency $\omega$, and the frequency $\omega$ is used in the transform. In particular,

$$
x_{d q 0}=T(\omega t) x_{a b c}
$$

Definition 4 (Common Direct-Quadrature Coordinates): As in [8], the direct-quadrature-zero coordinates in the common reference frame, denoted by the subscript $D Q 0$, are obtained when the Park transformation (1) is applied to a three-phase AC signal $x_{a b c}$ with frequency $\omega$, and the common reference frequency $\omega_{\text {com }}$ is used in the transform. In particular,

$$
x_{D Q 0}=T\left(\omega_{c o m} t\right) x_{a b c}
$$

Remark 2: Given $x_{a b c}$ as in Definition 1, the zero components of $x_{d q 0}$ and $x_{D Q 0}$ are zero. Hence $x_{d q}, x_{D Q}$ refer to the first two entries of $x_{d q 0}$ and $x_{D Q 0}$ respectively.

Definition 5 (Transformation from $d q$ to $D Q$ reference frame): As in [8], given $x_{d q}$, its transformation to the common $D Q$ reference frame is given as

$$
x_{D Q}=\mathcal{M}(\delta(t)) x_{d q}
$$

where $\mathcal{M}(\delta(t))=\left[\begin{array}{cc}\cos \delta(t) & -\sin \delta(t) \\ \sin \delta(t) & \cos \delta(t)\end{array}\right]$ and $\delta(t)$ is the deviation angle between the two reference frames.

\section{A. Grid-forming Inverter Model}

In this section we present the model of the inverter we will be considering for our control design. The assumptions used to obtain this model are given in Assumption 1 below.

Assumption 1:

- The DC-side is fast-reacting and equipped with sufficient energy reserves. The DC voltage is therefore stiff.

- Assuming a sufficiently high switching frequency (typically in the order of $10 \mathrm{kHz}$ or higher), the inverter switching process can be neglected.

- The power generated on the DC-side is supplied to the AC-side without switching losses.

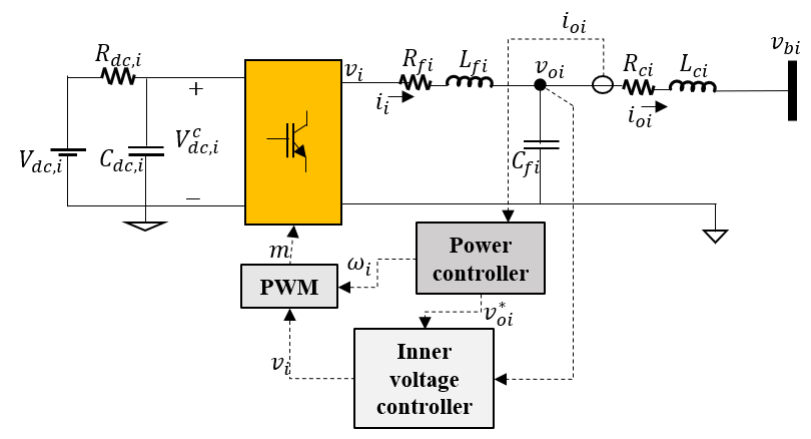

Fig. 1. The modelled DG grid-forming inverter block diagram.
As shown in Fig. 1, the inverter output voltage is filtered through an $L C$ filter to attenuate the high frequency components and harmonics generated by the switching process. The inverter is connected to the bus via a coupling connector. Following Assumption 1 the dynamics of the $L C$ filter and $L$ connector are described by the average model as follows:

$$
\begin{aligned}
\dot{i}_{i} & =-\frac{R_{f i}}{L_{f i}} i_{i}+\frac{1}{L_{f i}} v_{i}-\frac{1}{L_{f i}} v_{o i} \\
\dot{v}_{o i} & =\frac{1}{C_{f i}} i_{i}-\frac{1}{C_{f i}} i_{o i} \\
\dot{i}_{o i} & =-\frac{R_{c i}}{L_{c i}} i_{o i}+\frac{1}{L_{c i}} v_{o i}-\frac{1}{L_{c i}} v_{b i}
\end{aligned}
$$

where $R_{f i}, L_{f i}, C_{i} \in \mathbb{R}_{>0}$ are the resistance, inductance, and capacitance of the inverter $i L C$ filter; $R_{c i}, L_{c i} \in \mathbb{R}_{>0}$ are the connector resistance and inductance of inverter $i$; and $i_{i}, i_{o i}, v_{i}, v_{o i}$ are the input and output AC currents and voltages respectively of inverter $i$, and are signals of the form described in Definition 1. The bus voltage inverter $i$ is connected to is denoted as $v_{b i}$ and is a signal of the form described in Definition 1. Using the local frequency $\omega_{i}$ of inverter $i$, it is convenient to transform (5) to the local $d q$ coordinates [8]. This leads to the following equations:

$$
\begin{aligned}
\dot{i}_{d q i} & =\omega_{i} \mathcal{J} i_{d q i}-\frac{R_{f i}}{L_{f i}} i_{d q i}+\frac{1}{L_{f i}} v_{d q i}-\frac{1}{L_{f i}} v_{o d q i} \\
\dot{v}_{\text {odqi }} & =\omega_{i} \mathcal{J} v_{\text {odqi }}+\frac{1}{C_{f i}} i_{d q i}-\frac{1}{C_{f i}} i_{\text {odq } i} \\
\dot{i}_{\text {odqi }} & =\omega_{i} \mathcal{J} i_{\text {odqi }}-\frac{R_{c i}}{L_{c i}} i_{\text {odqi }}+\frac{1}{L_{c i}} v_{\text {odqi }}-\frac{1}{L_{c i}} v_{b d q i}
\end{aligned}
$$

where $i_{d q i}, i_{\text {odqi }}, v_{d q i}, v_{o d q i}, v_{b d q i}$ are signals that take values in $\mathbb{R}^{2}$ and are the local $d q$ currents and voltages of the form described in Definition 3, Remark 2. As in [8], it is convenient to interface the bus voltage $v_{b d q i}$ with the network via (4).

The deviation of individual inverter frequency $\omega_{i}$ from the common reference frame frequency $\omega_{\text {com }} \in \mathbb{R}$ is given as

$$
\dot{\delta}_{i}=\omega_{i}-\omega_{c o m}
$$

where $\delta_{i}$ is the deviation angle.

\section{B. Load and Transmission Line Models}

The load and transmission line models will be needed for the stability assessment of the overall system (section IV-A), and these models are presented in this section. We model the loads as balanced RL loads, and transmission lines as balanced $R L$ lines. The load connected to bus $i$, and the line connecting buses $i$ and $j$ are respectively described by (8) and (9).

$$
\begin{gathered}
\dot{i}_{\ell_{i}}=-\frac{R_{\ell_{i}}}{L_{\ell_{i}}} i_{\ell_{i}}+\frac{1}{L_{\ell_{i}}} v_{b i} \\
\dot{i}_{i j}=-\frac{R_{i j}}{L_{i j}} i_{i j}+\frac{1}{L_{i j}} v_{b i}-\frac{1}{L_{i j}} v_{b j}
\end{gathered}
$$

where $R_{\ell_{i}}, L_{\ell_{i}} \in \mathbb{R}_{>0}$ are the load resistance and inductance respectively, $i_{\ell_{i}}$ is the load current which is a signal of the form described in Definition $1 ; L_{i j}, R_{i j} \in \mathbb{R}_{>0}$ are respectively the line inductance and resistance; $i_{i j}, v_{b i}, v_{b j}$ are the line current and voltages at buses $i$ and $j$ respectively and are signals of the form described in Definition 1.

As in [8], it is convenient to transform (8) and (9) to the common $D Q$ reference frame. Hence their transformation to 
the common $D Q$ reference frame is obtained as

$$
\begin{gathered}
\dot{i}_{\ell D Q i}=\omega_{c o m} \mathcal{J} i_{\ell D Q i}-\frac{R_{\ell i}}{L_{\ell i}} i_{\ell D Q i}+\frac{1}{L_{\ell i}} v_{b D Q i} \\
\dot{i}_{D Q i j}=\omega_{c o m} \mathcal{J} i_{D Q i j}-\frac{R_{i j}}{L_{i j}} i_{D Q i j}+\frac{1}{L_{i j}} v_{b D Q i}-\frac{1}{L_{i j}} v_{b D Q j}
\end{gathered}
$$

respectively, where $i_{D Q i j}$ is the common $D Q$ line current, and $i_{\ell D Q i}, v_{b D Q i}$ are the common $D Q$ load current and voltage; $i_{D Q i j}, i_{\ell D Q i}, v_{b D Q i}$ are signals of the form described in Definition 4, Remark 2 and take values in $\mathbb{R}^{2}$.

\section{Conventional and Proposed Control Schemes}

\section{A. Conventional Control Scheme}

For active and reactive powers sharing, grid-forming inverters are usually equipped with conventional frequency and voltage controllers given as

$$
\begin{aligned}
\omega_{i} & =\omega_{n}-k_{p i} P_{i} \\
v_{o d i}^{*} & =V_{n}-k_{q i} Q_{i}, \quad v_{o q i}^{*}=0
\end{aligned}
$$

where $k_{p i}, k_{q i} \in \mathbb{R}_{>0}$ are the respective frequency and voltage droop gain; $\omega_{n}, V_{n} \in \mathbb{R}_{>0}$ are the respective nominal frequency and voltage, and $\omega_{i}, v_{\text {odqi }}^{*}$ are respectively the frequency and voltage set points. The inverter $i$ filtered active and reactive powers $P_{i}, Q_{i}$ are respectively obtained by measuring the active and reactive powers through a low-pass filter to attenuate high frequency noise. Their dynamics are

$$
\begin{aligned}
& \dot{P}_{i}=-\omega_{i c} P_{i}+\omega_{i c}\left(v_{\text {odi }} i_{\text {od } i}+v_{\text {oq }} i_{\text {oq }}\right) \\
& \dot{Q}_{i}=-\omega_{i c} Q_{i}+\omega_{i c}\left(v_{\text {oqi }} i_{\text {odi }}-v_{\text {odi }} i_{\text {oq } i}\right)
\end{aligned}
$$

where $\omega_{i c} \in \mathbb{R}_{>0}$ is the low-pass filter cutoff frequency. Finally, the PWM control signal is generated via the voltage (outer) and current (inner) controllers respectively:

$$
\begin{aligned}
& i_{d q i}^{*}=K_{P V i}\left(v_{o d q i}^{*}-v_{o d q i}\right)+K_{I V i} \int\left(v_{o d q i}^{*}-v_{o d q i}\right) d t \\
& v_{d q i}=K_{P C i}\left(i_{d q i}^{*}-i_{d q i}\right)+K_{I C i} \int\left(i_{d q i}^{*}-i_{d q i}\right) d t+\omega_{i} L^{\prime}
\end{aligned}
$$

where $K_{P V i}, K_{I V i} \in \mathbb{R}_{>0}$ are the proportional and integral gain of the voltage controller respectively, $K_{P C i}, K_{I C i} \in \mathbb{R}_{>0}$ are respectively the proportional and integral gain of the current controller.

\section{B. Proposed Control Scheme}

We modify (12) to eliminate the nonlinearities introduced into it by (13). This is achieved by employing the local directquadrature inverter output current $i_{\text {odqi }}$ as feedback to adapt the frequency and voltage. Since the current $i_{\text {odqi }}$ is obtained after the inverter $L C$ filter (see Fig. 1), it has low harmonic content which makes it a preferable choice. To enhance the controllers with disturbance rejection capability for noise free control of the frequency and voltage, $i_{\text {odqi }}$ is measured through a low-pass filter before applying it in the controllers.

Hence, the proposed controllers for the frequency and the voltage setpoint $v_{\text {odq }}^{*}$ are given by (15) and (16) respectively, together with the low pass filter dynamics (17).

$$
\begin{gathered}
\omega_{i}=\omega_{n}-m_{p i} \mathcal{I}_{o d i} \\
v_{o d q i}^{*}=\mathbb{I} V_{n}+\mathbf{e}_{\mathbf{2} i} \mathcal{I}_{\text {odq } i} \\
\dot{\mathcal{I}}_{\text {odq } i}=-\omega_{i c} \mathcal{I}_{\text {odqi }}+\omega_{i c} i_{o d q i}
\end{gathered}
$$

where $\mathbb{I}=\left[\begin{array}{ll}1 & 0\end{array}\right]^{\top}, \mathbf{e}_{2 i}=\left[\begin{array}{cc}-n_{q i} & 0 \\ 0 & n_{q i}\end{array}\right], m_{p i}, n_{q i} \in \mathbb{R}_{>0}$ are the frequency and voltage droop gains respectively; $\omega_{n}, V_{n} \in$ $\mathbb{R}_{>0}$ are the nominal frequency and voltage respectively; $\omega_{i}$ is the frequency, which is a signal that takes values in $\mathbb{R}$; $\omega_{i c} \in \mathbb{R}_{>0}$ is the filter cutoff frequency, and signal $\mathcal{I}_{\text {odqi }}$ is the filtered $i_{\text {odq } i}$.

Due to the phase lag introduced by integrators in a feedback loop, the use of two nested integrators will have the effect of leading to bad stability margins and oscillatory responses. Therefore, in our proposed design we replace conventional inner and outer control loops (14), with a single inner voltage controller. In particular, the error between the reference voltage $v_{o d q i}^{*}$ and inverter output voltage $v_{o d q i}$ is fed into a standard proportional-integral controller and is thus regulated to zero at steady state. More precisely, the control scheme is given by

$$
\begin{aligned}
\dot{\phi}_{d q i} & =v_{\text {odqi }}^{*}-v_{\text {odqi }} \\
v_{d q i} & =\Lambda_{P V i}\left(v_{o d q i}^{*}-v_{o d q i}\right)+\Lambda_{I V i} \phi_{d q i}
\end{aligned}
$$

where $\Lambda_{P V i}, \Lambda_{I V i} \in \mathbb{R}_{>0}$ are the proportional and integral gains respectively, and $\phi_{d q i}$ is the auxiliary state vector of the controller.

The remarks below describe key properties associated with our proposed controllers (15)-(18).

Remark 3: It should be noted that the frequency controller (15) provides active power sharing. In order to see this, consider a two-inverter system as shown in Figure 3 with each of the inverters (their parameters are denoted with subscripts 1,2 respectively) equipped with the frequency controller (15). If a steady state is reached where inverters 1 and 2 are synchronised, then $\omega_{1}^{*}=\omega_{2}^{*}$. By (15) this implies that $\omega_{n}-m_{p 1} \mathcal{I}_{o d 1}^{*}=\omega_{n}-m_{p 2} \mathcal{I}_{o d 2}^{*}$ which gives $m_{p 1} \mathcal{I}_{o d 1}^{*}=m_{p 2} \mathcal{I}_{\text {od }}^{*}$, and note that (17) yields $\mathcal{I}_{\text {odi }}^{*}=i_{\text {odi }}^{*}$ at steady state. Since the inverter output voltage does not deviate much, the relation $m_{p 1} \mathcal{I}_{o d 1}^{*}=m_{p 2} \mathcal{I}_{o d 2}^{*}$ gives a proportional sharing of the active power. If $m_{p 1}=m_{p 2}$, as chosen in our simulation, the active power is shared equally between the inverters irrespective of the load disturbances. This is evident in the simulation result.

Reactive power sharing is in general harder to guanrantee analytically and is investigated via simulations (section IV-B). In particular, these demonstrate that the proposed voltage controller (16)-(18) gives a better reactive power sharing at steady state than $(12 \mathrm{~b})$.

Remark 4: The negative feedback on the current $i_{\text {odq } i}$ and its combination with the droop gains in (16) implicitly provides a current limiting capability as it is explicitly offered by (14b). This is evident in the simulation results (section IV-B) where our controller offers a lower current compared to the conventional controller, and thus alleviates practical concerns associated with the inherent current limitation of inverters. This current limiting property of (16) makes it practically feasible to use a single loop inner voltage controller (18) (see also the related justification in [4], [13]), thus leading to an improved transient response as discussed below.

Remark 5: The key advantage of the proposed controllers (15)-(18), is that they offer improved stability margins and transient performance compared to the conventional controllers (14), (12). This is demonstrated in sections IV-A, IV-B via small signal stability analysis and simulations. As it has been discussed within this section this is something that is expected, as the additional phase lags due to the two nested integrators are avoided. 


\section{Performance of Proposed Control Scheme}

\section{A. Stability Assessment}

The proposed control scheme is compared to the conventional control scheme via the loci of the closed-loop dominant poles of the overall two-inverter system shown in Fig. (3). The small-signal model of two-inverter system equipped with the proposed controllers given by $(6),(7),(10),(11),(15)-(18)$, is compared to those equipped with the conventional controllers given by (6), (7), (10)-(14). Let $\Delta$ represent small deviations from the equilibrium point determined by simulation (see section IV-B), the small-signal state space model for the respective system set-up is described by

$$
\Delta \dot{x}=A \Delta x
$$

where $\Delta x=\operatorname{col}\left(\Delta x_{1}, \Delta x_{2}, \Delta i_{\ell D Q 1}, \Delta i_{\ell D Q 2}, \Delta i_{D Q 12}\right)$, $\Delta x_{1}, \Delta x_{2}$ take values in $\mathbb{R}^{2 n}$ and are the states of inverter 1 and 2 , and $A \in \mathbb{R}^{2 n \times 2 n}$ extracted accordingly. Due to limited space [8] can be consulted for a full description of obtaining (19).

Fig. 2 shows the loci of the dominant poles of the two set-ups with increasing droop gains. The blue " $*$ " are for the system equipped with our proposed controllers (15)-(18), while the red "O" are for those equipped the conventional controllers (12)-(14). It is evident that as the droop gains increase the complex dominant poles of the system equipped with the conventional controllers become unstable (when $k_{p i}=2.9 \times 10^{-3}$ ), while those with our proposed controllers still remain stable. Hence our proposed controllers offer better stability margins compared to the conventional controllers.

Remark 6: It should be noted that in the stability analysis presented in Fig. 2, the nominal gains of the conventional controllers have been numerically optimized so as to obtain the best possible transient response. This is done in order to ensure a fair comparison. It should also be noted that the difference in the stability properties of the two schemes is more fundamental, and is associated with the fact that one of the schemes avoids using double, nested integral action.

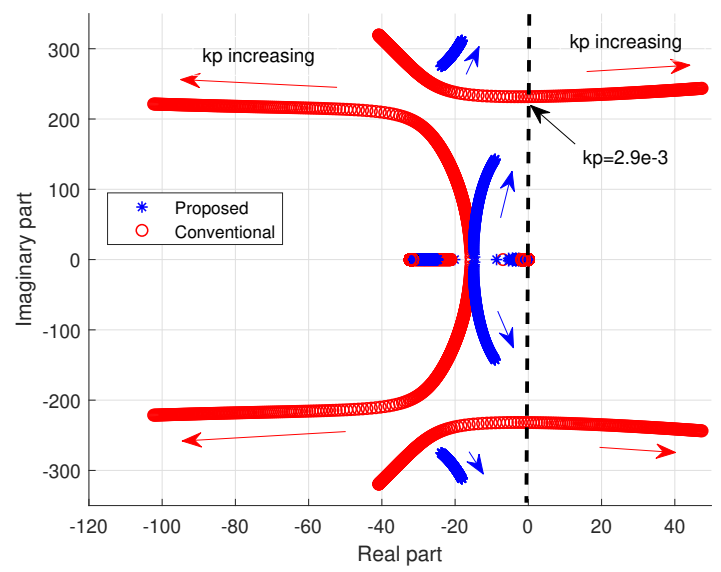

Fig. 2. Loci of the closed-loop dominant poles: blue " $*$ " for system equipped with the proposed controller; red " $\bigcirc$ " for the conventional controller.

\section{B. Time-Domain Simulation}

We compare the performance of the proposed controllers to the conventional controllers via simulations on a twoinverter test system (see Fig. 3) in MATLAB/Simscape Power Systems. To obtain realistic results the inverter models used in the simulations have PWM blocks which include the on/off actuation of the electronic switches. The system parameters are presented in Table I. The performance of the controllers is studied from the response of the system to step changes at the loads connected to the two buses. Figs. 4-7 respectively show the active power, reactive power, frequency and voltage responses of the test system for two step disturbances: a three-phase $R_{\ell_{1}}, L_{\ell_{1}}$ load switched on at bus 1 at $t=1.7 \mathrm{~s}$, and an equivalent load, $R_{\ell_{2}}, L_{\ell_{2}}$, switched off at bus 2 at $t=3.7 \mathrm{~s}$. As shown in Fig. 4, the current drooping of the proposed controller prevents excessive active power transfer in contrast to those with the conventional controller, which could mitigate the risk of inverter overcurrent. With the reduction of excessive active power transfer, Fig. 5 shows that the proposed controller offers reduced frequency deviations relative to the conventional controller. Some oscillations in frequency and active power observed with the conventional controller (see figures in situ in Figs. 4 and 5) do not occur in those with the proposed controller, hence preventing oscillations, and possibly instability between inverters during load changes. It is thus evident that the transient performance of the proposed controllers is improved with a smoother response. Fig. 6 shows that a better reactive power sharing is obtained for the system equipped with the proposed controller compared to the large reactive power sharing error with the conventional controller. This could prevent some DG inverters from being overstressed in supplying the required reactive power, hence improving system security. Fig. 7 shows that the output voltages of inverters with the proposed controller are better matched compared to those with the conventional controller. As shown in Fig. 8, the semiconductor peak current associated with the proposed controller is within the acceptable range and slightly lower than the conventional case. This shows that our controller provides current limiting capability, and thus alleviates the concern of using a single loop control.

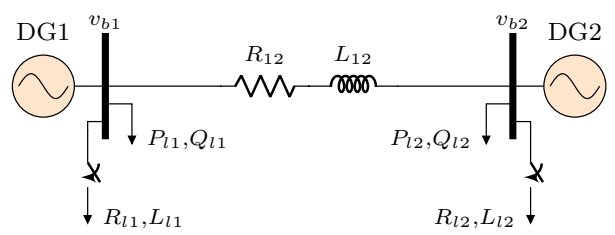

Fig. 3. Two-inverter test system.

TABLE I

INVERTER PARAMETERS

\begin{tabular}{ccc}
\hline$R_{f i}=0.05 \Omega$ & $L_{f i}=8 \mathrm{mH}$ & $C_{f i}=150 \mu \mathrm{F}$ \\
$R_{c i}=0.03 \Omega$ & $L_{c i}=7 \mathrm{mH}$ & $\omega_{n}=2 \pi(50) \mathrm{rad} / \mathrm{s}$ \\
$n_{q i}=0.0467$ & $m_{p i}=2 \pi(0.0295)$ & $w_{i c}=31.41 \mathrm{rad} / \mathrm{s}$ \\
$\Lambda_{P V i}=1, \Lambda_{I V i}=10$ & $K_{P V i}=5, K_{I V i}=5$ & $K_{P C i}=5, K_{I C i}=10$ \\
$k_{p i}=2 \pi(9.4) \times 10^{-5}$ & $k_{q i}=1.5 \times 10^{-4}$ & $P_{\ell_{1}}, P_{\ell_{2}}=1000 \mathrm{~W}$ \\
$Q_{\ell_{1}}, Q_{\ell_{2}}=100 \mathrm{Var}$ & $R_{\ell_{1}}, R_{\ell_{2}}=20 \Omega$ & $L_{\ell 1}, L_{\ell 2}=40 \mathrm{mH}$ \\
$V_{n}=311 \mathrm{~V}$ & $R_{12}=0.4 \Omega$ & $L_{12}=6 \mathrm{mH}$ \\
\hline
\end{tabular}

\section{CONCLUSION}

We have proposed a simple and effective frequency and voltage control scheme with desirable dynamic response, which eliminates nonlinearities in the generation of the PWM control signal, offers power sharing as well as current limiting capability, and improves the performance and stability of grid-forming inverters in a microgrid setting. This was demonstrated by a comparative study which examined the loci of the closed-loop dominant poles of the system with 

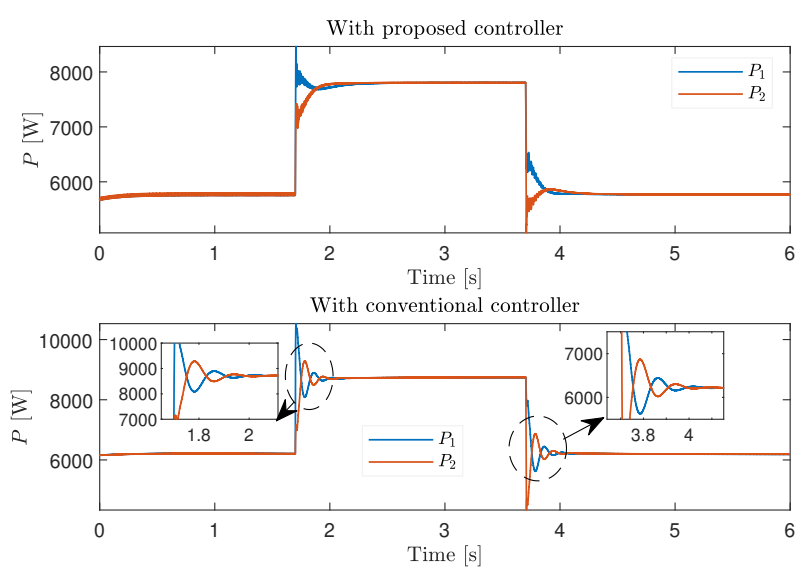

Fig. 4. System active power step responses

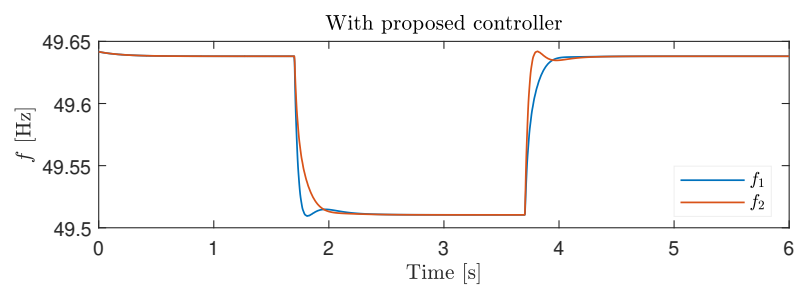

With conventional controller

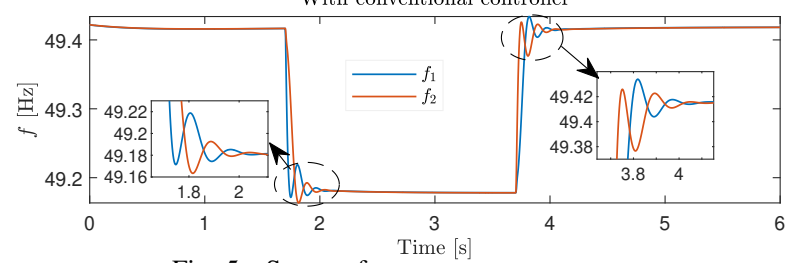

Fig. 5. System frequency step responses.
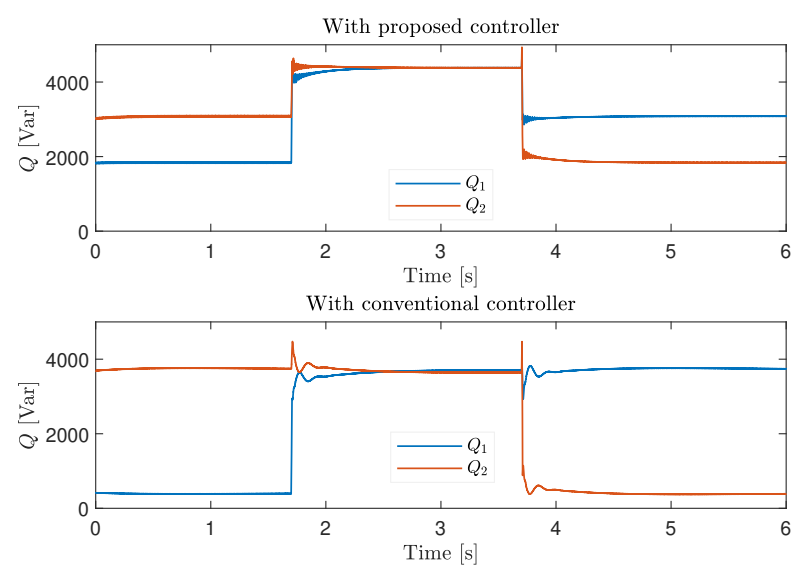

Fig. 6. System reactive power step responses.

increasing droop gains, and by simulation results in which a better transient performance was observed compared to the conventional hierarchical control scheme.

In future work we intend to design frequency and voltage controllers that help grid-forming inverters achieve passivity in the common reference frame. This will allow for the scalability of grid-forming inverters, by providing stability guarantees for interconnections of multiple grid-forming inverters in a general network topology.
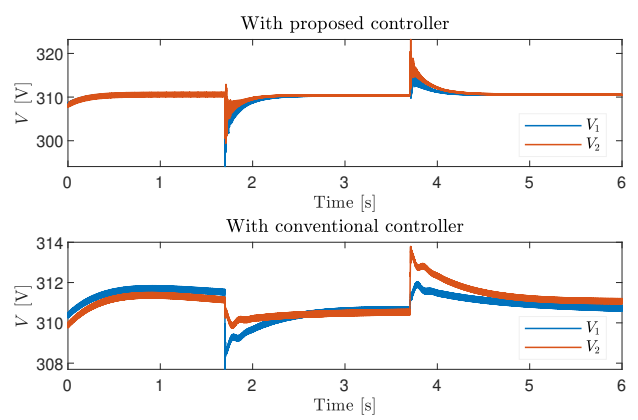

Fig. 7. System voltage step responses.
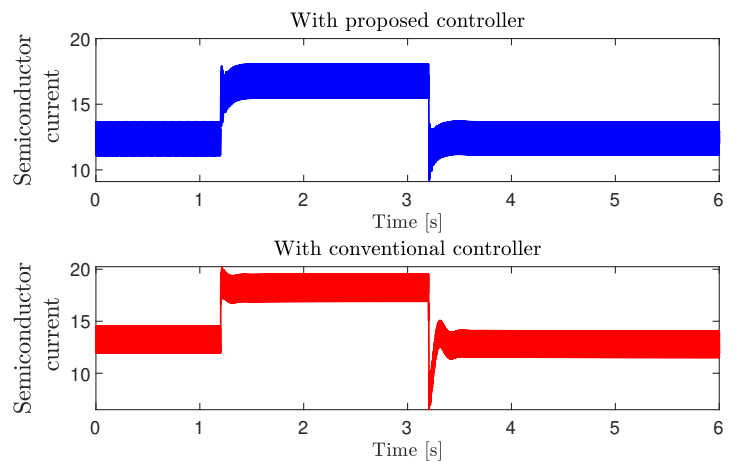

Fig. 8. Semiconductor current step responses.

\section{REFERENCES}

[1] M. C. Chandorkar, D. M. Divan, and R. Adapa, "Control of parallel connected inverters in standalone ac supply systems," IEEE Transactions on Industry Applications, vol. 29, no. 1, pp. 136-143, 1993.

[2] Y. Sun, X. Hou, J. Yang, H. Han, M. Su, and J. M. Guerrero, "New perspectives on droop control in ac microgrid," IEEE Transactions on Industrial Electronics, vol. 64, no. 7, pp. 5741-5745, 2017.

[3] J. M. Guerrero, L. G. de Vicuna, J. Matas, M. Castilla, and J. Miret, "Output impedance design of parallel-connected ups inverters with wireless load-sharing control," IEEE Transactions on Industrial Electronics, vol. 52, no. 4, pp. 1126-1135, 2005.

[4] X. Wang, Y. W. Li, F. Blaabjerg, and P. C. Loh, "Virtual-impedancebased control for voltage-source and current-source converters," IEEE Transactions on Power Electronics, vol. 30, no. 12, pp. 7019-7037, 2015.

[5] S. Curi, D. Grob, and F. Dörfler, "Control of low-inertia power grids: A model reduction approach," in 2017 IEEE 56th Annual Conference on Decision and Control (CDC), 2017, pp. 5708-5713.

[6] J. Aracil and F. Gordillo, "On the control of oscillations in dc-ac converters," in IEEE 2002 28th Annual Conference of the Industrial Electronics Society. IECON 02, vol. 4, 2002, pp. 2820-2825 vol.4.

[7] L. A. B. Torres, J. P. Hespanha, and J. Moehlis, "Power supply synchronization without communication," in 2012 IEEE Power and Energy Society General Meeting, 2012, pp. 1-6.

[8] N. Pogaku, M. Prodanovic, and T. C. Green, "Modeling, analysis and testing of autonomous operation of an inverter-based microgrid," IEEE Transactions on Power Electronics, vol. 22, no. 2, pp. 613-625, 2007.

[9] A. Bidram and A. Davoudi, "Hierarchical structure of microgrids control system," IEEE Transactions on Smart Grid, vol. 3, no. 4, pp. 1963-1976, 2012.

[10] F. Katiraei, R. Iravani, N. Hatziargyriou, and A. Dimeas, "Microgrids management," IEEE Power and Energy Magazine, vol. 6, no. 3, pp. 54-65, 2008.

[11] F. Milano, F. Dörfler, G. Hug, D. J. Hill, and G. Verbič, "Foundations and challenges of low-inertia systems (invited paper)," in 2018 Power Systems Computation Conference (PSCC), 2018, pp. 1-25.

[12] Y. Ojo, M. Benmiloud, and I. Lestas, "Frequency and voltage controllers for three-phase grid-forming inverters," under review, 2019.

[13] X. Wang, P. C. Loh, and F. Blaabjerg, "Stability analysis and controller synthesis for single-loop voltage-controlled vsis," IEEE Transactions on Power Electronics, vol. 32, no. 9, pp. 7394-7404, 2017. 